\title{
Ambulatory Antibiotic Prescribing for Children with Pneumonia After Publication of National Guidelines: A Cross-Sectional Retrospective Study
}

\author{
Nicole M. Poole - Daniel J. Shapiro - Matthew P. Kronman • \\ Adam L. Hersh
}

Received: September 20, 2019 / Published online: November 27, 2019

(C) The Author(s) 2019

\begin{abstract}
Introduction: National guidelines published in 2011 recommend amoxicillin as first-line treatment for non-hospitalized children with community-acquired pneumonia (CAP). We aimed to understand visit rates, antibiotic selection, and factors associated with amoxicillin prescribing for children with CAP since guideline publication.
\end{abstract}

Methods: We performed a cross-sectional retrospective study of patients aged 90 days-18 years with an outpatient clinic or emergency department (ED) visit from 2008 to 2015 using the National Ambulatory Medical Care Survey and the National Hospital Ambulatory Medical Care Survey ED data files, respectively. We estimated the incidence rates of ambulatory CAP visits, examined time trends

Enhanced Digital Features To view enhanced digital features for this article go to https://doi.org/10.6084/ m9.figshare.10033262.

N. M. Poole $(\bowtie) \cdot$ M. P. Kronman

Pediatric Infectious Diseases, University of

Washington, Seattle, WA, USA

e-mail: Nicole.Poole@childrenscolorado.org

D. J. Shapiro

Pediatrics, Boston Children's Hospital, Boston, MA, USA

A. L. Hersh

Pediatric Infectious Diseases, University of Utah,

Salt Lake City, UT, USA in antibiotics prescribed at CAP visits, and determined factors independently associated with first-line guideline-recommended antibiotic prescribing using multivariable logistic regression, including patient age, setting, and US census region.

Results: From 2008 to 2015, there were an estimated 1.5 million [95\% confidence interval (CI) 1.3-1.7 million] pediatric CAP visits annually. Amoxicillin was prescribed in 23\% (95\% CI $18-30 \%)$, azithromycin was prescribed in $47 \%$ (95\% CI 41-54\%), and cephalosporins were prescribed in $26 \%$ (95\% CI $21-31 \%)$ of antibiotic visits for CAP. There were no significant differences in annual CAP visits or prescribing by antibiotic class since guideline publication. Amoxicillin prescribing was significantly less likely in visits by older children, aged 5-18 years, [adjusted odds ratio (aOR) 0.22, 95\% CI $0.10-0.49$ ] compared to visits by younger children aged 90 days- 4 years with CAP. Compared with the Northeast, amoxicillin prescribing was significantly lower in the Midwest (aOR $0.35,95 \%$ CI 0.13-0.98) and South (aOR 0.23, 95\% CI 0.08-0.63). Azithromycin prescribing was significantly more likely in visits to EDs (aOR 1.46, 95\% CI 1.07-1.98) compared to physician offices.

Conclusion: Despite national guideline recommendations, amoxicillin prescribing for CAP in outpatient settings is low and azithromycin remains the predominant antibiotic prescribed, highlighting the need for dedicated antibiotic 
stewardship efforts in ambulatory settings.Keywords: Antibiotic; Antibiotic stewardship; Azithromycin; Community acquired pneumonia; Guideline; Pediatrics

\section{Key Summary Points}

\section{Why carry out this study?}

National guideline recommendations published in 2011 recommend amoxicillin as the first-line antibiotic for the treatment of pediatric community acquired pneumonia (CAP) in ambulatory settings.

A comprehensive evaluation of antibiotic prescribing for ambulatory pediatric patients with CAP for the entirety of the pediatric population since national guideline publication is lacking.

This study describes the trends in visit rates and antibiotic prescribing, as well as factors associated with first-line guideline prescribing, for pediatric CAP in ambulatory settings since national guideline publication.

\section{What was learned from the study?}

Since the publication of national guidelines, amoxicillin prescribing in pediatric CAP has remained low (20\% in $2008-2011$ vs. $28 \%$ in 2012-2015, $p=0.15$ ); azithromycin remains the primary antibiotic prescribed (47\% in $2008-2011$ vs. $48 \%$ in $2012-2015$, $p=0.91)$, particularly in older children aged 5-18 years (OR 2.27; 95\% CI 1.26-4.07, compared to younger children aged 90 days -4 years) and in pediatric visits to emergency departments (OR 1.46; 95\% CI 1.07-1.98, compared to office visits).

Despite national guideline recommendations, amoxicillin prescribing for CAP in outpatient settings is low and azithromycin remains the predominant antibiotic prescribed for all age groups, highlighting the need for dedicated antibiotic stewardship efforts in ambulatory settings.

\section{INTRODUCTION}

In 2011, the Infectious Disease Society of America and the Pediatric Infectious Disease Society published national practice guidelines for the management and treatment of children older than 3 months of age with communityacquired pneumonia (CAP). The guideline recommends against routine antimicrobial therapy for preschool children with CAP, as viruses are the primary etiology of pneumonia in this age group. For suspected bacterial CAP in outpatient settings, amoxicillin is recommended as the first-line antibiotic to target Streptococcus pneumoniae, and macrolide antibiotics are recommended as an alternative for patients with symptoms consistent with CAP secondary to atypical pathogens (e.g. Mycoplasma pneumoniae) or with severe beta-lactam allergy [1].

Prior to national guideline publication, antibiotics were prescribed in about $70 \%$ of outpatient visits for CAP, of which amoxicillin was prescribed at $14 \%$ and macrolides (primarily azithromycin) were prescribed at $34 \%$ of all pediatric ambulatory visits $[2,3]$. Previous work has demonstrated no change in first-line antibiotic prescribing for children aged 1-6 years old with CAP in outpatient settings since the publication of national guideline recommendations [3]. However, a comprehensive evaluation of antibiotic prescribing in CAP for the entirety of the pediatric population since national guideline publication is lacking.

We aimed to understand whether changes in visit rates and antibiotic selection for children aged 90 days -18 years with CAP in ambulatory settings occurred after the publication of the national guideline recommendations. We also aimed to explore the factors associated with first-line guideline prescribing for CAP in outpatient settings since the guideline publication.

\section{METHODS}

We performed a cross-sectional retrospective study of patients aged 90 days- 18 years with an outpatient clinic or emergency department (ED) visit from 2008 to 2015 using the National 
Ambulatory Medical Care Survey (NAMCS) and the National Hospital Ambulatory Medical Care Survey (NHAMCS) ED data files, respectively. NAMCS and NHAMCS are surveys using a multistage probability design conducted annually by the National Center for Health Statistics (NCHS) to provide nationally representative data about visits for ambulatory care in both physician offices (NAMCS) and EDs (NHAMCS) [4]. The data in the surveys include patient demographics, diagnoses associated with the visit based on International Classification of Diseases, 9th revision (ICD9) codes, and medications prescribed. Additional information includes the United States geographic region of the visit based on US Census Regions (Northeast, South, Midwest and West) and insurance status.

Patients with complex chronic conditions [5] and those hospitalized or referred from the office to an ED were excluded. Pneumonia visits were defined as visits with an ICD9 code for pneumonia (480.0-2, 480.8-9, 481, 482.0, 482.30-2, 482.41-2, 482.83, 482.89-90, 483.8, $484.3,485,486,487.0)$ in any of the first three diagnosis fields [6].

We estimated the incidence rates of ambulatory CAP visits, examined time trends in antibiotics prescribed at CAP visits, and determined factors independently associated with first-line guideline-recommended antibiotic prescribing using multivariable logistic regression, including patient age, setting (office vs. ED) and US census region. Civilian non-institutionalized population data from the U.S. Census Bureau [7] were used to determine antibiotic visits per 1000 U.S. population. Population data from the U.S. Census Bureau are not available by month or day of age; therefore, we corrected the population data for patients $<1$ year of age by a factor of 90/365 to account for patients $<90$ days of age who were excluded from this study. We assessed trends in the average annual CAP rate over time using logistic regression with 2-year increments (2008-2009, 2010-2011, 2012-2013, and 2014-2015) as the predictor variable. Antibiotic prescribing proportions and comparisons before and after guideline publication were compared using Chi square tests. We performed statistical analyses using STATA $14^{\circledR}$ (STATA, USA). Estimates were not calculated if based on $<30$ visits or if the relative standard error was $>0.3$, as recommended by NCHS standards for reliability and precision [8]. Ninety-five percent confidence intervals (CI) were calculated for all estimates. Significance was considered at 2-sided $p$ value $\leq 0.05$. The datasets used in this analysis are de-identified and publicly available and thus determined non-human subjects research.

\section{RESULTS}

From 2008 to 2015, there were an estimated 1.5 million (95\% CI 1.3-1.7 million) pediatric CAP visits, or 19.8 (95\% CI 17.3-22.3) CAP visits per 1000 children, annually. An estimated 68\% (95\% CI $64-73 \%$ ) of the ambulatory CAP visits were to office clinics; the remaining visits were to EDs. There was no significant trend $(p=0.45)$ in the average annual rate of CAP visits over the study period.

Antibiotics were prescribed in $77 \%$ (95\% CI $72-81 \%$ ) of outpatient visits for CAP, and there was no significant difference in overall antibiotic prescribing in the years prior to the guideline publication (2008-2011) compared to the years following the guideline publication $(2012-2015 ; 77 \%$ vs. 78\%, $p=0.85)$. When an antibiotic was prescribed, amoxicillin was prescribed in 23\% (95\% CI 18-30\%), azithromycin was prescribed in $47 \%$ (95\% CI $41-54 \%$ ), and cephalosporins were prescribed in $26 \%$ (95\% CI 21-31\%) of CAP visits overall. Azithromycin was the predominant antibiotic prescribed in offices and EDs at CAP visits in all age groups (Table 1). There was no significant difference in amoxicillin, azithromycin, or cephalosporin prescribing in the years prior to the guideline publication compared to the years following the guideline publication (Table 1).

Amoxicillin prescribing was significantly less likely in visits by older children aged $5-18$ years [adjusted odds ratio (aOR) 0.22, 95\% CI $0.10-0.49$ ] compared to visits by younger children aged 90 days -4 years with CAP (Table 2). Compared with the Northeast, amoxicillin prescribing was significantly lower in the Midwest (aOR 0.35, 95\% CI 0.13-0.98) and South (aOR 


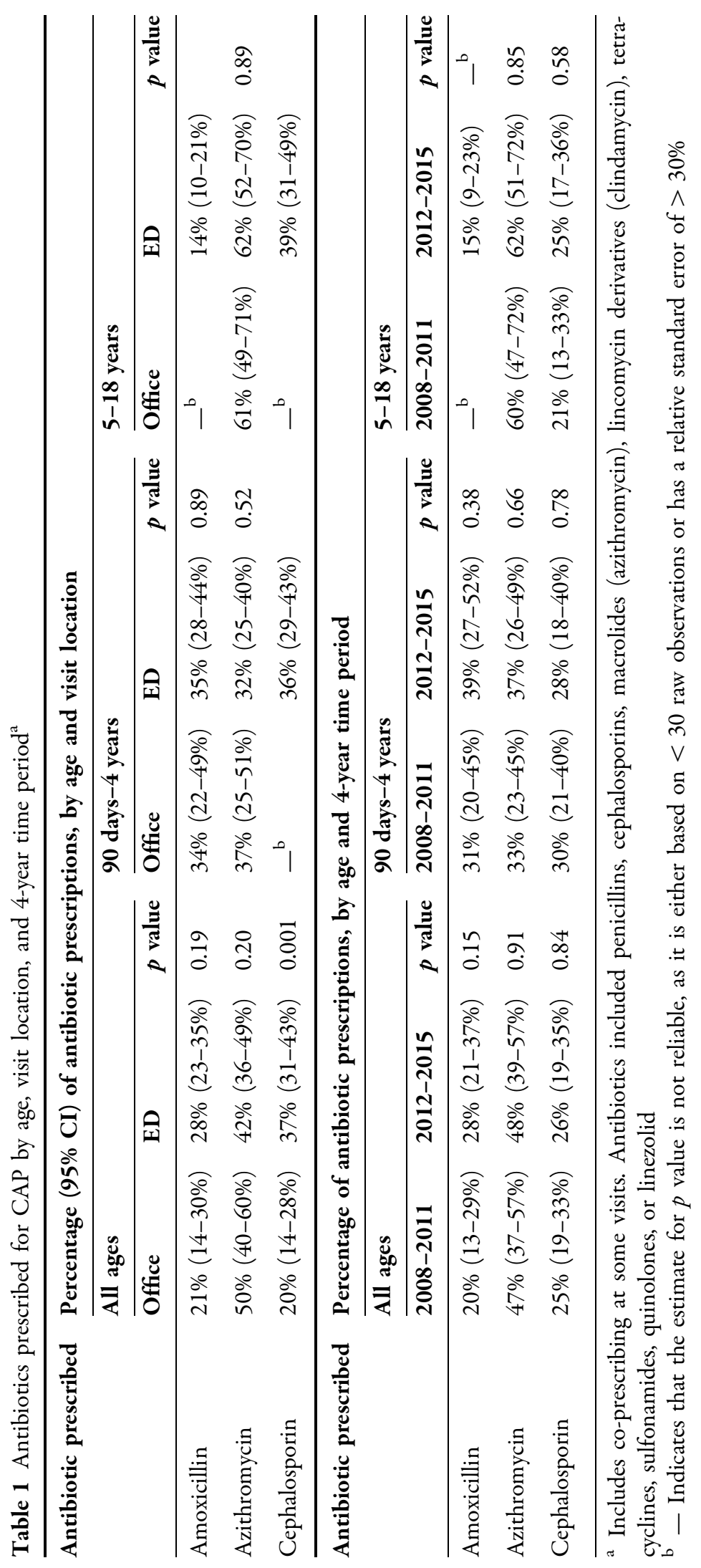


0.23, 95\% CI 0.08-0.63; Table 2). Azithromycin prescribing was significantly more likely in visits to EDs (aOR 1.46, 95\% CI 1.07-1.98) compared to physician offices (Table 2).

\section{DISCUSSION}

This study describes the incidence of and antibiotic prescribing for pediatric CAP in outpatient settings nationally since the publication of national guideline recommendations. The rates of CAP diagnoses and antibiotic prescribing for CAP have been unchanged since the national guideline publication. Despite guideline recommendations to prescribe amoxicillin as the first-line antibiotic choice for children with CAP, there has been no significant increase in amoxicillin prescribing since publication, and azithromycin remains the predominant antibiotic prescribed for children of all ages with CAP. Older children and children residing in the Midwest and South are less likely to receive amoxicillin for CAP compared to younger children and children living in the Northeast. Children who visit EDs are more likely to receive azithromycin compared to children who visit a physician office.
Previous work that investigated antibiotic prescribing for children aged 1-6 years with CAP similarly found no differences in antibiotic prescribing overall or antibiotic class when comparing prescribing before and after guideline publication [3]. We found that younger children (aged 90 days-4 years) were prescribed azithromycin and amoxicillin at similar rates. However, when the entire population was expanded to all pediatric patients aged 90 days- 18 years, we found that azithromycin was prescribed more frequently, and significantly more so in older children (aged 5-18 years) compared to younger children and when the visit occurred at an ED compared to an office setting. The majority of pediatric visits to EDs occur nationally at non-pediatric facilities, and previous work has shown that children are prescribed unnecessary antibiotics, particularly azithromycin, more frequently in non-pediatric EDs compared to pediatric EDs [9]. It is possible that children in this study received azithromycin more frequently in EDs due to lower prevalence of pediatric-trained physicians at the majority of EDs nationally. Additionally, although patients admitted to the hospital were excluded, patients could have presented to EDs with more concerning symptoms compared to physician offices, prompting physicians in EDs

Table 2 Factors associated with amoxicillin and azithromycin antibiotic prescribing for CAP

\begin{tabular}{lll}
\hline Characteristic & \multicolumn{2}{l}{ Adjusted odds ratio (95\% CI) } \\
\cline { 2 - 3 } & Amoxicillin monotherapy & Azithromycin monotherapy \\
\hline Age 5-18 years (vs. 90 days-4 years) & $0.22(0.10-0.49)$ & $2.27(1.26-4.07)$ \\
Male sex (vs. female) & $0.86(0.46-1.62)$ & $1.22(0.74-2.01)$ \\
White race (vs. nonwhite) & $1.33(0.69-2.56)$ & $0.84(0.45-1.58)$ \\
Region (vs. Northeast) & & \\
Midwest & $0.35(0.13-0.98)$ & $0.75(0.31-1.82)$ \\
South & $0.23(0.08-0.63)$ & $0.59(0.24-1.47)$ \\
West & $0.58(0.22-1.54)$ & $0.72(0.30-1.72)$ \\
Non-private insurance (vs. private) & $1.40(0.65-3.01)$ & $0.81(0.61-1.09)$ \\
ED (vs. office) & $0.85(0.49-1.47)$ & $1.46(1.07-1.98)$ \\
\hline
\end{tabular}


to treat for atypical pneumonia. However, the estimated rate of pediatric pneumonia from Mycoplasma pneumoniae is estimated to be as low at $8 \%$, and we found that azithromycin was prescribed to nearly $50 \%$ of all CAP visits [10]. Other etiologies of atypical pneumonia, such as Chlamydia pneumoniae, are much less prevalent $(<1 \%$ of pediatric CAP hospitalizations) in the US [11].

Azithromycin is inappropriate treatment for the majority of pediatric ambulatory CAP diagnoses, as $S$. pneumoniae is much more likely to be resistant to azithromycin than to amoxicillin [10]. This study was not designed to assess clinical outcomes; therefore, we are unable to determine whether there was a difference in patient outcomes according to the antibiotic prescribed. However, the pathogenicity and clinical significance of M. pneumoniae is unclear and atypical pneumonia is often self-limited [10], which makes azithromycin treatment for atypical pneumonia of unclear benefit.

It is unknown why physicians favor azithromycin over amoxicillin for the treatment of CAP, and additional studies are needed to further explain this phenomenon. Factors that might contribute to the over-prescription of azithromycin include the convenience of azithromycin dosing and duration, over-estimation of the prevalence and morbidity of $M$. pneumoniae, and the over-diagnosis of penicillin allergy in children. The risk of over-prescribing azithromycin includes increasing resistance to $S$. pneumonia and S. pyogenes, the most common bacterial causes of acute respiratory tract infections in children. Other harmful effects of antibiotics include auto-immune diseases, other infectious diseases such as Clostridium difficile, and obesity [12].

It is possible the national guideline recommendations for the treatment of CAP have not been widely referenced or utilized because clinical practice is slow to change. It is estimated to take 17 years for scientific discovery to enter daily clinical practice [13]. However, inpatient settings have shown a significant increase in first-line guideline prescribing for hospitalized children with CAP since the publication of the national guideline recommendations [14]. Hospitals with individualized plans for guideline implementation were significantly more likely to prescribe first-line antibiotics to children with CAP [14], highlighting the importance of dedicated antibiotic stewardship (AS) efforts in guideline implementation.

Finally, previous work has found geographic variation in unnecessary antibiotic and azithromycin prescribing in outpatient settings [15]. This study adds to this work by showing similar geographic variability in first-line guideline-recommended antibiotic prescribing for CAP. Geographic variations in the rate of azithromycin prescribing could be partially explained by disease prevalence; however, cultural differences nationally surrounding antibiotic prescribing could be playing a significant role in prescribing trends. Future studies are needed to better understand why this geographic discordance exists in antibiotic prescribing.

This study has several limitations. First, patient allergies or antibiotics prescribed at previous visits, which can influence antibiotic prescribing, are unavailable. However, it is expected that a true penicillin allergy and previous medication failure would contribute to a small percentage of indicated azithromycin prescriptions [10]. Second, patient-level data including vital signs and physical exam findings are unavailable, limiting our ability to assess the severity of illness, which could influence prescribing practices. Third, visits for CAP were identified based on three diagnosis codes available in the NHAMCS dataset, which were not directly linked to medications mentioned in the visit. It is therefore possible that the indication of CAP for the antibiotic mentioned could be misclassified.

\section{CONCLUSIONS}

We found that, although amoxicillin is the firstline antibiotic recommended for ambulatory children with CAP, there has been no significant increase in amoxicillin prescribing since publication of the national guideline recommendations in 2011. In contrast to our findings for ambulatory visits, previous work has shown 
that, for children hospitalized with CAP, firstline narrow-spectrum antibiotic use had an absolute increase of $27 \%$ after national guideline publication, with greater increases among hospitals that underwent local implementation efforts [16]. AS programs have primarily dedicated efforts to improve inpatient antibiotic prescribing, and have less presence in outpatient and ED settings [17]. This study highlights that national guidelines alone are inadequate, and that dedicated ambulatory AS efforts are necessary to improve the quality of antibiotic prescribing for CAP in ambulatory settings.

\section{ACKNOWLEDGEMENTS}

We thank the participants of the study. The findings and conclusions in this report are those of the authors and do not necessarily represent the official position of the Centers for Disease Control and Prevention.

Funding. This work was supported by the Centers for Disease Control and Prevention (CDC) [IPA\# 1708452 MOD2 to A. L. H.]. No funding was received for the publication of this article.

Authorship. All named authors meet the International Committee of Medical Journal Editors (ICMJE) criteria for authorship for this article, take responsibility for the integrity of the work as a whole, and have given their approval for this version to be published.

Disclosures. Nicole M. Poole, Daniel J. Shapiro, Matthew P. Kronman and Adam L. Hersh have no personal, financial, commercial, or academic conflicts of interest. Nicole M. Poole conducted this work at the University of Washington and her current affiliation is at the University of Colorado School of Medicine, Aurora, CO, USA. Adam L. Hersh is a member of the journal's Editorial Board.

Compliance with Ethics Guidelines. The data sets used in this analysis are de-identified and publicly available and thus determined non-human subjects research.
Data Availability. The datasets analyzed during the current study are publicly available in the NAMCS and NHAMCS repository, https:// www.cdc.gov/nchs/ahcd/datasets_documentation_related.htm.

Open Access. This article is distributed under the terms of the Creative Commons Attribution-NonCommercial 4.0 International License (http://creativecommons.org/licenses/ by-nc/4.0/), which permits any noncommercial use, distribution, and reproduction in any medium, provided you give appropriate credit to the original author(s) and the source, provide a link to the Creative Commons license, and indicate if changes were made.

\section{REFERENCES}

1. Bradley JS, Byington CL, Shah SS, et al. Executive summary: the management of community-acquired pneumonia in infants and children older than 3 months of age: clinical practice guidelines by the Pediatric Infectious Diseases Society and the Infectious Diseases Society of America. Clin Infect Dis. 2011;53(7):617-30.

2. Kronman MP, Hersh AL, Feng R, Huang YS, Lee GE, Shah SS. Ambulatory visit rates and antibiotic prescribing for children with pneumonia, 1994-2007. Pediatrics. 2011;127(3):411-8.

3. Florin TA, Byczkowski T, Gerber JS, Ruddy R, Kuppermann N. Diagnostic testing and antibiotic use in young children with community-acquired pneumonia in the United States, 2008-2015. J Pediatric Infect Dis Soc. 2019.

4. Centers for Disease Control and Prevention National Center for Health Statistics. Ambulatory Health Care Data. https://www.cdc.gov/nchs/ahcd/ new_ahcd.htm. Accessed Mar 31, 2017.

5. Feudtner C, Feinstein JA, Zhong W, Hall M, Dai D. Pediatric complex chronic conditions classification system version 2: updated for ICD-10 and complex medical technology dependence and transplantation. BMC Pediatr. 2014;14:199.

6. Williams DJ, Shah SS, Myers A, et al. Identifying pediatric community-acquired pneumonia hospitalizations: accuracy of administrative billing codes. JAMA Pediatr. 2013;167(9):851-8. 
7. United States Census Bureau. Monthly population estimates for the United States: April 1, 2010 to December 1, 2018.

8. McCaig LF, Burt CW. Understanding and interpreting the National Hospital Ambulatory Medical Care Survey: key questions and answers. Ann Emerg Med. 2012;60(6):716-721.e711.

9. Poole NM, Shapiro DJ, Fleming-Dutra KE, Hicks LA, Hersh AL, Kronman MP. Antibiotic prescribing for children in United States Emergency Departments: 2009-2014. Pediatrics. 2019;143(2):e20181056.

10. Blyth CC, Gerber JS. Macrolides in children with community-acquired pneumonia: panacea or placebo? J Pediatric Infect Dis Soc. 2018;7(1):71-7.

11. Jain S, Williams DJ, Arnold SR, et al. Communityacquired pneumonia requiring hospitalization among US children. N Engl J Med. 2015;372(9): $835-45$.

12. Vangay P, Ward T, Gerber JS, Knights D. Antibiotics, pediatric dysbiosis, and disease. Cell Host Microbe. 2015;17(5):553-64.
13. Westfall JM, Mold J, Fagnan L. Practice-based research-"Blue Highways" on the NIH roadmap. JAMA. 2007;297(4):403-6.

14. Williams DJ, Edwards KM, Self WH, et al. Antibiotic choice for children hospitalized with pneumonia and adherence to national guidelines. Pediatrics. $2015 ; 136(1): 44-52$.

15. Fleming-Dutra KE, Demirjian A, Bartoces $M$, Roberts RM, Taylor TH Jr, Hicks LA. Variations in antibiotic and azithromycin prescribing for children by geography and specialty-United States, 2013. Pediatr Infect Dis J. 2018;37(1):52-8.

16. Williams DJ, Hall M, Gerber JS, et al. Impact of a national guideline on antibiotic selection for hospitalized pneumonia. Pediatrics. 2017;139(4): e20163231.

17. Mistry RD, Newland JG, Gerber JS, et al. Current state of antimicrobial stewardship in children's hospital emergency departments. Infect Control Hosp Epidemiol. 2017;38(4):469-75. 